Derecho y Realidad

Vol.14 Núm. 27 • Enero Junio de 2016

Págs. 165-185 - ISSN:1692-3936

\title{
La internacionalización de la educación superior y su vulneración como derecho. Análisis dentro de las políticas públicas 1992-2014. Caso UPTC*
}

\author{
The Internationalization of the Higher \\ Education and its Infrigement as a \\ Fundamental Right. Analysis within Public \\ Policies 1992-2014. The UPTC case
}

Carlos Andrés Lizarazo Aparicio***

\section{Resumen}

El objetivo de la presente investigación es analizar desde el punto de vista de las políticas públicas colombianas la internacionalización de la educación y su vulneración como derecho. Para ello se determinan las directrices de los organismos internacionales del orden económico en la formulación de las

Fecha de aceptación: 08 de octubre de 2015

Concepto de recepción: 14 de octubre de 2015

Fecha de aprobación: 23 de octubre de 2015

* El presente artículo es resultado del proyecto de investigación terminado, La internacionalización de la educación superior y su afectación como derecho. Análisis dentro de las políticas públicas 19922014. Caso UPTC.

** Licenciado en Filosofía, egresado de la Universidad Pedagógica y Tecnológica de Colombia de Tunja. Estudiante de Maestría en Derechos Humanos en la misma Universidad. Docente del Servicio Nacional de Aprendizaje (SENA) regional Tunja. calizarazo67@misena.edu.co 
politicas públicas educativas en Colombia; se establece la forma como se afecta el derecho a la educación por parte de estas y se elabora una propuesta de política pública que garantice el derecho a la educación en este país. La vulneración del derecho a la educación por parte de las políticas educativas en Colombia se ha evidenciado especialmente desde el año 1992, con la Ley 30, en los diversos planes de desarrollo, porque los programas de gobierno de cada mandatario se han dirigido a disminuir los recursos que aportaba el Estado, incluyendo los subsidios con que se beneficiaban muchos estudiantes de bajos recursos. Respecto a la internacionalización, producto de la globalización, la UPTC intenta emular el ambiente que se impone en centros educativos de otros países desarrollados, en cuanto a calidad educativa, evaluación, portafolio de programas, entre otros.

\section{Palabras clave}

Derechos fundamentales, globalización, gratuidad, internacionalización, neoliberalismo, políticas, privatización.

\section{Abstract}

This paper aims to analyze from the point of view of the Colombian public policies the internationalization of education and its effects as a right. For that purpose international organizations guidelines of the economic order are determined in the formulation of educational public policies in Colombia; it is established the effects on the right to education and it is prepared a draft of public policy that guarantees the right to education in Colombia. Infrigement of the right to education by educational policies in Colombia has been evidenced specially since 1992, with Law 30, in the various development plans because government programs of each ruler were directed to reduce the State resources, including subsidies that many low-income students benefited. Regarding internationalization, product of globalization, the UPTC tries to emulate the atmosphere of other developed countries in terms of educational quality, portfolio of programs, among others.

\section{Keywords}

Fundamental rights, globalization, gratuity, internationalization, neoliberalism, policies, privatization. 


\section{Introducción}

Este trabajo analiza desde la perspectiva legal y política las dinámicas, la concepción, las implicaciones y los retos del proceso de internacionalización, liderado por las instituciones de educación superior (IES), a fin de establecer lineamientos para entender y viabilizar elementos legales para el desarrollo de la internacionalización de la educación superior. El proceso de internacionalización permite observar las diferentes consideraciones en torno a la influencia del mismo en las dinámicas de la educación superior.

Teniendo en cuenta que el proceso de internacionalización toma elementos que son propios de fenómenos socioeconómicos como la globalización y la liberalización económica, este estudio aborda la problemática del tema a partir de los cambios normativos y de política pública, para integrar el campo de la educación al sector económico y productivo (Van der Wende, 2001). De esta manera, el estudio da cuenta del proceso de internacionalización de la educación superior colombiana, que implica el análisis de la evolución del proceso normativo y de política pública para el manejo de esta educación en el país.

El proceso de internacionalización, poco regulado y escasamente acompañado por políticas públicas, ha significado que actores de la educación superior como entidades de gobierno del sector educativo, instituciones, así como asociaciones y redes universitarias diseñen y ejecuten acciones para la internacionalización de la educación superior de manera independiente, con una notoria falta de articulación (Misas, 2004; Vásquez, 2011). En la mayoría de los casos se presenta una duplicidad de esfuerzos y mayor inversión de recursos, que en términos generales significan para el país una menor respuesta al proceso global y poco éxito de las estrategias (Asociación Colombiana de Universidades [ASCUN], 2008).

\section{Metodología}

La presente investigación se orientó por el enfoque mixto, siguiendo las exigencias que la Maestría en Derechos Humanos determina en los lineamientos para la elaboración del proyecto de investigación. Así mismo, el trabajo monográfico se fundamentó bajo el campo "Línea 2. Políticas públicas en derechos humanos", para ello se llevó a cabo "el análisis crítico de políticas públicas específicas teniendo como base el derecho humano desde el cual se producen" (Maestría de Derechos Humanos), las cuales corresponden a la educación superior pública, que se imparte en la Universidad Pedagógica y Tecnológica de Colombia (UPTC) (Estudio de caso). El tipo de investigación que se utilizó en el desarrollo del presente trabajo fue el estudio de caso, mediante el cual se analizó desde el punto de vista de las políticas públicas colombianas la internacionalización de la educación y su vulneración como derecho, catalogada por Yin (1994) como investigación empírica. Como instrumentos se utilizaron las fichas bibliográficas y las de contenido para obtener 
la información de las fuentes consultadas. La población objeto, en este caso, son los estudiantes de la Universidad Pedagógica y Tecnológica de Colombia (UPTC) que adelantan estudios en los diversos programas de pregrado, posgrado, doctorados y a distancia.

\section{Resultados y discusión}

\section{Investigación}

\subsection{Descripción del problema}

Una de las grandes problemáticas que se han venido presentando en Colombia desde hace algún tiempo, ha sido la desigualdad en la educación y la vulneración de los derechos de niños y jóvenes por la mala calidad de la enseñanza y la investigación, un nivel de producción científica muy bajo, unas políticas de Estado inadecuadas y una gran carencia de recursos. Ninguna universidad colombiana aparece en el ranking de Shanghai de las mejores 500 universidades del mundo, ni de las 400 universidades top del Times Higher Education, los dos rankings de mayor reputación en el mundo.

Se ha observado en los últimos años el crecimiento de la educación privada y cómo ha bajado el nivel y la calidad en la educación pública, un factor clave para el fenómeno que se está presentando actualmente. Esto se debe a las políticas de educación que el gobierno ha adoptado, haciendo más grande la brecha entre clases sociales, ya que los menos favorecidos solo deben conformarse con una tecnología o una carrera técnica, que son a las cuales pueden acceder; en otras palabras no se ha permitido la equidad o justicia educativa. Lo que hace pensar que la educación no es un derecho, como lo dice la Constitución Política de Colombia, educación para todos, si no que se ha convertido en un negocio, que ha favorecido a algunas instituciones privadas, que han tomado fuerza gracias a la tecnología y el mundo de las telecomunicaciones.

Este es un problema relacionado con las instituciones, pues son pocas las que tienen desarrollada una política de internacionalización que involucre, desde su construcción y formulación hasta su ejecución, a todos los actores, y que responda al plan y a la estrategia general institucional. Hay escasos desarrollos frente a las propuestas de contar con una mirada integral de la internacionalización.

La responsabilidad de las instituciones de educación superior con el contexto se aborda en forma aislada desde la investigación, docencia, extensión o proyección social; no se trata desde una política integral institucional, lo cual disminuye los beneficios de la internacionalización. Esto hace imprescindible analizar la educación en Colombia, las políticas educativas que se han acogido y qué medidas ha desarrollado el gobierno para garantizar la equidad en la educación y cómo ha sido la intervención en los procesos educativos en las instituciones oficiales. 
Por lo cual, es preciso definir si los contenidos en la educación tendrán la calidad correspondiente a la capacidad de pago, del estudiante o cliente, según se determine si es un derecho humano o un bien transable, dados por intereses alejados de la pedagogía, inclinados al mercado y la internacionalización de la economía. Es necesario reflexionar sobre la trascendencia que debe tener la equidad educativa en relación con la justicia social, política, cultural y económica nacional.

\subsection{Formulación del problema}

¿Qué directrices establecidas por organismos internacionales del orden económico afectan el derecho a la educación superior en Colombia?

\subsection{Justificación}

Llama la atención y pone el dedo en la llaga sobre una problemática grave que está afectando a la educación superior en Colombia y en el mundo, por lo que es oportuno recordar que la internacionalización integral es el compromiso, confirmado a través de las acciones, para introducir una perspectiva internacional y comparativa en la enseñanza, en la investigación y en las misiones de la educación superior.

Es lamentable observar que la educación superior pública está desapareciendo en Colombia, para darle paso a una serie de concesiones al capital, al sector privado, para que asuman el control y la administren en el país. En el país son muy pocos los jóvenes que pueden acceder a una educación superior, pues deben pagar altas sumas de dinero por un semestre en universidades privadas o, lo que es aun más grave, en las públicas. Se convirtió la educación en un proceso selectivo que profundiza las diferencias entre clases sociales y que no favorece postulados tan importantes como la inclusión social y la democratización de la educación.

Es en esa medida que resulta fundamental fortalecer el papel del Estado como educador y con una perspectiva clara de servicio y no como un negocio. También es de vital importancia ejercer controles para evitar que los abusos del sector privado sean encubiertos, así como quienes hacen uso de ellos. Casos como el de la Universidad San Martín, que resultó siendo un negocio tramposo y un saqueo contra los colombianos, no deben volver a suceder en el país.

Por tanto, es necesario que el Estado recupere su misión y su visión de regulador fiscal de la educación colombiana, y que esté plenamente comprometido con la educación de los niños y jóvenes, pero recuperando su vocación de servicio y rechazando su papel de mercader o de simple espectador, teniendo presente que la educación es la base de la sociedad. Por ello, cada vez se debe inculcar, tanto en el docente como en el estudiante, la importancia que tiene la calidad de la educación y lo afortunado que es quien la recibe. Hay que revisar lo de la Superintendencia de la Educación Superior. 


\subsection{Objetivos}

\subsubsection{Objetivo general}

Analizar desde el punto de vista de las políticas públicas colombianas la internacionalización de la educación superior y su vulneración como derecho.

\subsubsection{Específicos}

- Determinar las directrices establecidas por los organismos internacionales del orden económico en la formulación de las políticas públicas educativas de la educación superior en Colombia.

- Exponer la vulneración del derecho a la educación superior por parte de las políticas educativas en Colombia.

- Establecer una propuesta de política pública que garantice el derecho a la educación superior en Colombia.

\subsection{Marco referencial}

\subsubsection{Estado del tema y marco teórico}

Para Cañón (2005), en su análisis sociológico de la educación superior en Colombia y Latinoamérica, el fenómeno de la internacionalización de esta y los procesos de globalización, siempre han existido, aunque con otras denominaciones, gracias a la influencia del imperialismo, que ha ejercido y ejerce influencia en los ámbitos culturales y políticos, donde la influencia de los "opresores", ha sido marcada, incluso en la educación. Es así como la universidad "moderna y nacional" tiene su génesis en Europa, luego es traída a América con la fidelidad en sus lineamientos, que el autor de la referencia denomina como "el primer fenómeno de la educación superior como tal" (Cañón, 2005).

Así mismo, Cañón reconoce que la modernidad ejerció notoria influencia en las universidades, gracias al carácter laico que se les imprime y a la masificación que se gesta en las mismas, como una necesidad para formar ciudadanía y amoldarse a las necesidades que exigía la industria, a través de sus avances y revoluciones. Sin embargo, hay que reconocer la continua resistencia a las exigencias de acreditación y "evaluación de pares internacionales", catalogadas como un irrespeto para su identidad (Cañón, 2005).

En la pugna que genera en las instituciones de educación superior su integración al ambiente internacionalizador actual, es importante considerar que la educación pública en algunos países latinoamericanos todavía mantiene un ápice de subsidio para una microminoría, porque se ha gestado y aplicado el mercantilismo en la determinación de las matrículas, lo que las convierte en privatizadas, en sentido 
inverso a la calidad que imparten, lo que es cuestionado por parte de algunas instituciones (OCDE, 2012; Ascún, 2008, 2014), debido a las limitaciones que enfrentan.

Ante el fenómeno de la privatización, la internacionalización de la educación superior se cataloga como una necesidad y fenómeno "socioeconómico global", que conlleva retos y riesgos, pero también oportunidades que pueden ser aprovechadas para fortalecer las IES, las cuales deben tomar la decisión como instituciones y organizaciones que son, a través de la participación de los diferentes estamentos que las conforman, con una mirada crítica constructiva, bajo la dirección de los profesionales de mayor capacidad que integran las diversas unidades de investigación y estudio.

Para la Asociación Internacional de Universidades, la internacionalización de la educación superior es una necesidad que contribuye al desarrollo de la humanidad:

(...) la mejora de la calidad de la enseñanza, el aprendizaje y la investigación; mayor compromiso con los actores nacionales, regionales y mundiales; mejor preparación de los estudiantes como ciudadanos globales y nacionales; acceso a los estudiantes a programas que no están disponibles o que se encuentran escasos en su país de origen; mayores oportunidades para la mejora del profesorado y, a través de la movilidad, la disminución del riesgo de "endogamia" académica. (Asociación Internacional de Universidades, 2012)

Quiere decir lo expuesto por los analistas de la Asociación Internacional de Universidades (2012), que estas no pueden quedarse estancadas y anquilosadas en el tiempo, porque deben actualizarse y competir con las demás que están ubicadas en sus países y fuera de ellos, especialmente, los industrializados o desarrollados, lo que permite capacitar a los egresados con calidad, actualidad y eficiencia, que a la vez, facilita el acceso a la continua demanda y exigencias de las empresas multinacionales, que son las que continuamente requieren personal formado, acorde con las exigencias de la globalización.

A fin de mostrar la necesidad de la internacionalización universitaria, la Organización para la Cooperación y el Desarrollo Económico (OCDE) analizó e identificó los problemas más destacados de la educación superior colombiana, en la equiparación con otros países, entre los cuales están: a) movilidad académica, b) problemas relacionados con el manejo de una lengua extrajera, y c) carencia o deficiencia en las políticas (Organización para la Cooperación y el Desarrollo Económico [OCDE], 2012).

En el caso de la internacionalización, en lo que respecta a la movilidad económica, el país afronta múltiples "cuellos de botella" de diferente orden, difíciles de solucionar, si las actitudes y acciones del Estado no se realizan con celeridad, responsabilidad 
y apoyo financiero. Estas debilidades las afrontan los estudiantes, pero también los docentes, lo que imposibilita brindar una educación con calidad acorde a los lineamientos y estándares internacionales, formación que a la vez contribuye con aporte de profesionales, según las exigencias del mercado, que además les ofrece un salario según la exigencia del mundo globalizado y sus multinacionales.

Por su parte, en las conclusiones publicadas por el MEN de los Diálogos regionales sobre la Educación Superior, se manifiesta la ausencia de una política de bilingüismo en Colombia que trace metas a medio y largo plazo (Ministerio de Educación Nacional, 2013). En este caso, tres instituciones reconocidas nacional e internacionalmente, afirman enfáticamente que las instituciones que tienen a cargo la educación superior en Colombia presentan serias falencias en el manejo de lenguas extranjeras, donde es marcada la ausencia de políticas de bilingüismo, tanto en herramientas como en la correspondiente implementación. Razón por la cual se incumple con los estándares internacionales, en este campo, y son factores que conducen a que los egresados del país estén imposibilitados para competir con los de otras universidades a nivel continental y global, y por eso no pueden acceder a las exigencias de este mercado.

Los dos problemas citados anteriormente (movilidad académica internacional y manejo de lengua extranjera por parte de las IES) se sustentan en la carencia de políticas del orden nacional e institucional, porque la internacionalización no se ha asumido en forma integral, asimismo, existen serios cuestionamientos al diseño metodológico y puesta en práctica bajo criterios globalizadores (currículo), de la educación superior, lo que muestra de igual manera, ausencia de criterios básicos de la internacionalización.

Las características anteriores indican la radiografía de las fortalezas, debilidades, amenazas y oportunidades de las instituciones de educación superior en América Latina, pero especialmente en Colombia, que se enfrentan al devenir de la globalización para crecer y ofrecer una educación con calidad y competitividad o quedarse en el atraso y acentuar el subdesarrollo que aqueja a los países donde se encuentran ubicadas.

En ese orden de ideas, para Roth:

(...) las políticas de privatización, la configuración de un Sistema de Educación Superior y adecuación a los lineamientos de las grandes agencias internacionales como el Banco Mundial y el Fondo Monetario Internacional tienen una cierta continuidad en Latinoamérica y, por tanto, después de la "Primera Reforma" habría sólo una "Segunda Reforma" que se ha venido implementando desde los años ochenta del siglo pasado hasta el presente (Roth, 2011). 
Roth (2011) afirma que son solo dos reformas las que ha enfrentado la educación superior en América Latina, porque elimina la tercera, al ser una sucesión de las dos anteriores, cuyas acciones están incluidas en los primeros procesos. En ese afán por internacionalizarse, las instituciones de educación superior catalogan como una prioridad hacerlo en contravía de los efectos que tengan estas decisiones en un gran sector de la población colombiana. Y, en ese orden de ideas, la Universidad Nacional de Colombia destina una de sus grandes líneas de acción para esa tarea (la Línea 6 internacionalización). Uno de los programas de esta universidad atiende directamente la "Movilidad e intercambio profesoral y estudiantil".

Los problemas que en este caso se resaltan, se centran en que la educación que se imparte a distancia o virtual a través de la internacionalización, no está acorde con las exigencias de la globalización y muchas veces es inferior en calidad a programas presenciales que ofrece la misma universidad en su sede principal (Castell, 1996).

En el caso de que las universidades opten por no satisfacer la demanda que exige el mundo global, las reduce al ambiente local, porque sacrifica posibilidades de vida que son valiosas; sin embargo, las condiciones de competencia local, regional, nacional e internacional, están latentes ante un juego que es difícil de descifrar, por la oferta de programas que no son acordes en su formación y calidad, lo que conduce a graduar profesionales con capacidad limitada y debilitada que no pueden acceder a la demanda de las empresas multinacionales (De Wit, 2013).

El panorama que se observa en las IES refleja una problemática que, en la búsqueda de soluciones se encontró con otras de mayor envergadura, debido a las exigencias del mundo global, donde se refleja la competencia del capitalismo, problemas que se ahondan con el atraso en que se sumen las universidades y que empeora la calidad de la educación y, lógicamente, la formación de los egresados; lo cual significa que cada vez hay una mayor oferta de profesionales que no tienen oportunidad en el mercado laboral, circunstancia que incide negativamente en las cifras de empleo y en la situación social de las familias.

La situación expuesta llevó a que con un mayor número de estudiantes producto de la creciente demanda, aumentaran las obligaciones de las universidades públicas, frente a unos ingresos estatales que no mantuvieron la misma dinámica, y esa inequidad generó en estos centros la necesidad de actualizarse para, al menos, mantenerse con recursos propios y competir en desigualdad con las privadas. Es por ello, que las primeras optan por mercantilizarse, aumentando los costos de los semestres en pregrado, pero enfatizando en los posgrados, especializaciones y doctorados, adicionando además el cobro por la venta de servicios académicos, sacrificando, en este caso, las oportunidades que tenían los más pobres para acceder a la educación superior (Ramírez, 2012). 
En la disyuntiva surgida por la competencia entre la educación superior pública, frente a la privada que la aventajaba numéricamente, la primera optó por la búsqueda de alternativas para su financiación diferentes al Estado, que limitó su apoyo y en consecuencia se perdió la dominación hegemónica que mantenían las estatales, lo que generó un aumento en el portafolio de programas, derechos y servicios, al igual que el surgimiento de nuevas que entraron a disputarse el mercado existente (Chiroleu, 2005).

Lo anterior quiere decir que el cambio en el funcionamiento del sector público y el privado obedece más a las exigencias de los organismos multilaterales de crédito (FMI, BM y BID), a quienes el país adeuda cuantiosas sumas en dólares, que se desbordan en capital e intereses cuando esta moneda sube de valor y que para garantizar el pago periódico exigen condiciones que se orientan más al incremento de impuestos y a la rentabilidad de los servicios que presta el Estado, razón por la cual se privatizan, como es el caso de la educación, con la consecuente debilitación de lo público.

En ese orden de ideas, para las IES es insostenible su situación porque mientras el escaso aporte del Estado se mantiene en las mismas cifras de décadas anteriores, los costos para atender la creciente demanda resultan imposibles de cubrir con la precariedad de los recursos, razón por la cual, se requirió la incorporación de fuentes de financiamiento, por la mercantilización de servicios, otros derechos, el aumento de los semestres para pregrado, pero con mayor intensidad para posgrados y doctorados, a la par que se eliminaron subvenciones y becas otorgadas a personas de escasos recursos para contribuir con su sostenimiento (manutención y costo de los semestres) mientras estudiaban.

Lo que se observa en los comentarios de Chiroleu (2005) es que el punto de equilibrio de las IES demuestra que con los aportes estatales es imposible cubrir los costos, generándose pérdidas acumuladas, que le dan inestabilidad a las universidades, razón por la cual acuden a la mercantilización y lógicamente, a la privatización. Esa privatización lleva de igual manera a hacer lo propio con el conocimiento y el trabajo académico, que se cataloga como una producción de "mercancías cognitivas" y se enfrenta con la que se genera en las universidades públicas. También por la venta de ese nuevo bien, que a la vez se traduce en oportunidades de ingreso, dejando de ser una institución estatal para dar nacimiento a una empresa.

Esto quiere decir que las personas con menor capacidad económica deben conformarse con no ingresar a la universidad para adelantar estudios superiores, porque el Estado les está atendiendo otras necesidades sociales, y para ello, las familias también deben esforzarse por hacer su aporte económico y el resto lo aporta el capital privado a través de préstamos que, en este caso, sería con intereses y condiciones onerosas. 
Los argumentos expresados, recogidos de los autores que no están de acuerdo con la privatización y la mercantilización de la universidad pública, son valederos, porque es una realidad que cada vez más la familia de menores recursos ve alejadas las posibilidades de que sus miembros adelanten estudios superiores por el alto costo de estos debido a la eliminación de múltiples beneficios que facilitaban el acceso a estos centros y por la insuficiencia de ingresos. La connotación en este caso es doble, porque la única oportunidad para algunos es adelantar estudios de pregrado, lo cual no es garantía de acceso al mercado laboral, porque existen profesionales que les compiten con varios posgrados, especializaciones o doctorados, razón por la cual la única opción es aceptar un cargo, de ser posible, ajeno a su perfil y con un ingreso que no cubre sus expectativas.

En ese orden de ideas, en ese ambiente de mercantilización, las universidades públicas y privadas, después de que los estudiantes culminan su estudio de pregrado, a través de la difusión en los medios de comunicación, promueven la idea de no realizar trabajo de grado y para ello le ofrecen que solo pague un semestre de posgrado o especialización y con el cumplimiento de este requisito se gradúa.

La advertencia que hacen Altbach et al. (2010) es que algunos programas catalogados como pilares para la formación en valores, identidad, ciudadanía y democracia, de los ciudadanos y lógicamente de la familia, carecen de la demanda suficiente para mantenerlos, razón por la cual están condenados a desaparecer, porque las necesidades están demarcadas por la globalización y por las empresas multinacionales, que exigen el tipo de profesional que requieren para mantener los crecientes avances de la ciencia y la tecnología.

La propuesta de Nussbaum (2010) se centra en que la universidad pública latinoamericana tiene el compromiso de formar profesionales y ciudadanos que sean críticos, sensibles, pero sobre todo, que sean ciudadanos del mundo que contribuyan al trabajo en común.

\subsection{Diseño metodológico}

La presente investigación se orientó a través del enfoque mixto, siguiendo las exigencias que la Maestría en Derechos Humanos determina mediante Lineamientos para la elaboración del proyecto de investigación. Este método, como lo expresan Johnson y Onwuegbuzie (2004), favorece de igual manera la triangulación, entre teoría, autores de escritos y realidad, a través del análisis desde el punto de las políticas públicas colombianas, la internacionalización de la educación y su vulneración como derecho; de igual manera, facilita corroborar la información obtenida e interpretarla (Driessnack, 2007). 


\subsubsection{Línea de investigación}

Este trabajo monográfico se fundamentó bajo el campo "Línea 2. Políticas públicas en derechos humanos", para ello se abordó "el análisis crítico de políticas públicas específicas teniendo como base el derecho humano desde el cual se producen" (Maestría de Derechos Humanos), las cuales corresponden a la educación superior pública, que se imparte en la Universidad Pedagógica y Tecnológica de Colombia (UPTC) (Estudio de caso).

\subsubsection{Tipo de investigación}

La investigación que se utilizó fue el estudio de caso, mediante el cual se analizó desde el punto de vista de las políticas públicas colombianas la internacionalización de la educación y su vulneración como derecho, catalogada por Yin, como investigación empírica (Yin, 1994)

\subsubsection{Instrumentos}

Se utilizaron las fichas bibliográficas y la de contenido para obtener la información de las fuentes consultadas.

\subsubsection{Población y muestra}

La población objeto son los estudiantes de la Universidad Pedagógica y Tecnológica de Colombia (UPTC) que adelantan estudios en los diversos programas de pregrado, posgrado, doctorados y a distancia.

\subsection{Análisis e interpretación de resultados}

En la vulneración del derecho a la educación superior por parte de las políticas educativas en Colombia, uno de los aspectos más relevantes para el Banco Mundial, respecto a los diagnósticos que ha realizado en la educación superior en América Latina, es la politización, que cada vez se encarga de carcomer los escasos recursos que asigna el Estado y los que los padres de familia, con gran esfuerzo, se ven abocados a pagar por el mismo despilfarro, ineficiencia y caos administrativo que las aqueja (Banco Mundial, 1998).

Para nadie es un secreto quiénes son los políticos (desde ministros, senadores, representantes a la cámara, gobernadores, alcaldes, diputados, concejales y muchos de estos ex) que tienen a la Universidad Pedagógica y Tecnológica de Colombia sumida en una crisis desde hace muchos años, con el agravante de que los sindicatos que existen de igual manera son responsables de que la educación haya dejado de ser un derecho para convertirse en una mercancía, porque en este último caso generaciones de familias se han lucrado y siguen haciéndolo con los cargos que 
han disfrutado gracias al legado de sus ancestros, amañados y amarrados por las mangualas que allí se ciernen (Bank for Reconstruction and Development \& The World Bank, 2012).

Por otra parte, como lo afirma el Banco Mundial, para atender esa desmesurada demanda surgieron las ofertas de carreras a distancia, virtuales, posgrados y especializaciones y doctorados de diferente orden, adecuándose mínimamente a las exigencias internacionales para brindar programas de calidad, especialmente en las zonas más alejadas del país.

De la misma manera, el Banco Mundial enfatiza que las reformas a la educación superior, originadas en la década de los noventa, gracias a la influencia del neoliberalismo, dieron lugar a la violación del derecho a la educación por la mercantilización de la misma, porque "el mercado entró a las universidades públicas y transformó los conceptos de ciudadanía estudiantil, democracia institucional y formación para el desempeño de funciones públicas" (International Association of Universities, 2012).

\subsubsection{Antecedentes de la privatización en la Universidad Pedagógica y Tecnológica de Colombia}

Al comparar los parámetros que desde el año 1994 viene aplicando la Universidad Pedagógica y Tecnológica de Colombia para el cobro de semestres en las diferentes facultades y programas, se observa que estos son los mismos en cuanto a la cantidad de salarios mínimos mensuales vigentes, lo que da lugar a una variación creciente leve con el incremento que realiza el Estado cada año. Lo mismo ocurrió entre los años 2013 a 2016, en los que se mantienen dichos parámetros, a diferencia de los que se cobran por declaración de renta, que son una minoría.

La afirmación de la referencia se demuestra al consultar los 26 acuerdos (dos de liquidación mediante declaración de renta y 24 de incremento en los diferentes programas). Situación similar a la que ocurre con los programas presenciales se observa en los programas a distancia, donde se han aprobado ocho acuerdos.

En cuanto a los derechos complementarios cobrados a los estudiantes en cada semestre, se tienen en cuenta el concepto, la norma que los contempla y la tendencia de las variables utilizadas para cuantificarlas, en donde en la mayoría prima el salario mínimo mensual legal vigente, aumento anual según el monto autorizado por el Gobierno (2013-2016).

En el año 2009, las directivas de la UPTC consideraron prudente realizar un estudio que permitiese a partir de allí tener parámetros confiables para las estimaciones del valor de la matrícula de sus estudiantes y para ello contrataron con la Universidad Nacional lo que se denomina "Metodología para el cálculo del valor de las matrículas 
en la Universidad Pedagógica y Tecnológica de Colombia" (Universidad Nacional, 2009), que se convirtió en el pilar para la privatización de la educación pública en la región.

Por otra parte, para comparar la tendencia de número de estudiantes que ingresaron a las universidades de educación públicas, gracias al programa "Ser Pilo Paga", las diferencias son considerables respecto a lo captado por las privadas $(51.4 \%$ de las matrículas de los pilos, para siete universidades privadas). Lo alarmante es que las privadas recibieron el $98,4 \%$ de la totalidad del presupuesto $(\$ 218.872 .001 .623)$ y las oficiales, solo el 1.6\% (\$3.558.894.334), lo que se justifica por el bajo costo del semestre de las públicas, el cual se fundamenta en las tablas determinadas para los estudiantes y no con el real promedio por estudiante.

Al observar el total de recursos recibidos a marzo de 2016 del programa "Ser Pilo Paga" por la UPTC, comparada con nueve universidades oficiales, esta ocupa el primer lugar con $\$ 803.440 .795$, mientras que en los dos últimos se ubican la de Caldas (\$55.171.346) y la de Cauca (\$41.986.000). Y le siguen en orden descendente la de Pereira, Nacional, UIS, Manizales, Cartagena, Valle y Antioquia, con cifras que se pueden catalogar de sorprendentes porque el valor reconocido se fija según el promedio que tenga el centro universitario.

Respecto al número de estudiantes matriculados del programa "Ser Pilo Paga", la Universidad Nacional ocupa el primer puesto, siguiendo en orden descendente la del Valle, UIS, Pereira, UPTC (5. ${ }^{\circ}$ puesto), Antioquia, Caldas, Cartagena, Cauca y Manizales. En cuanto al valor promedio de matrícula reconocido por cada estudiante del programa "Ser Pilo Paga", en el 2016, en el primer puesto se destaca la Universidad de Manizales (\$5.408.697) y en segundo lugar, la UPTC (\$1.046.285), en tercer lugar la de Pereira (\$645.388.371), cuarto lugar la Nacional $(\$ 378.762)$ y quinto la UIS (\$411.972).

Las cifras anteriores (marzo de 2016) muestran en cierta forma un panorama de los recursos que cobran por matrícula las universidades públicas, reflejados por el valor promedio que el Estado reconoció por estudiante matriculados que resultaron favorecidos con el programa "Ser Pilo Paga". A pesar de la difícil situación económica que afronta, la UPTC resultó mejor posicionada que las nueve restantes, pero llama la atención que a la Universidad de Manizales le reconocieron \$5.408.697 por cada matrícula, cifra que se supone le permite obtener los recursos suficientes para su funcionamiento y una utilidad que está bastante alejada de las demás oficiales.

\subsubsection{Educación presencial}

Como se pudo observar a partir del año 2010, gracias al estudio realizado, se acentuó la privatización de la educación superior en la UPTC, logrando percibir un 
mayor monto de recursos con los cobros de los semestres de posgrado, maestrías y doctorados.

Uno de los aspectos por resaltar es que a los estudiantes de pregrado y a distancia, en cierta forma, se les desmotiva para que elaboren trabajos de monografías o tesis, ya sea porque no se les asigna director (regularmente este no tiene tiempo, para asesorarlo), porque no le nombran jurados, y en cambio, los "obligan" a pagar un semestre de posgrado y cumpliendo dicho requisito se gradúan, situación que ha llevado a que la demanda de estos programas sea cada vez mayor, no solo por parte de estudiantes de la UPTC, sino de otras universidades (públicas y privadas) locales y aledañas.

A la par con lo anterior, como nunca antes había ocurrido, el número de graduados en diferentes facultades es cada vez mayor, los cuales se enfrentan a una realidad local y regional, exceso de oferta de profesionales en diferentes áreas, y a una escasa o casi nula demanda.

- Pregrados por Facultad. En el ámbito de las Ciencias Agropecuarias (2); Ciencias Básicas (4); Ciencias de la Educación (11); Ciencias Económicas y Administrativas (3); Ciencias de la Salud (3); Derecho y Ciencias Sociales (1); Ingeniería (10).

- El valor de cada semestre en el primer rango es de $\$ 2.413 .093,00$, la anualidad $\$ 4.826 .185,00$ y los cuatro semestres o dos años de $\$ 10.090 .204,00$. Respecto al segundo rango, el semestre oscila entre $\$ 2.757 .820$,oo y $\$ 4.136 .730,00$ es de $\$ 6.894 .550$ hasta $\$ 8.273 .460$.oo y la anualidad de $\$ 13.789 .100$,oo a $\$ 16.546 .920$,oo y por último, los 190 créditos por salario mínimo diario legal vigente equivalen respectivamente a $\$ 8,728.500$ (año 1), \$9.339.497,oo.

- Doctorados. La Universidad Pedagógica y Tecnológica de Colombia ofrece siete doctorados, para adelantar en cuatro años, cuyos montos van desde 5 hasta 11,5 SMMLV por semestre. El valor más elevado de los cuatro años de doctorado es de \$70.890.516,oo (23 SMMLV en los primeros dos años y 15 SMMLV en los tres años restantes. En ese orden de ideas, el doctorado más barato es de $\$ 30.611 .410,00$ (5 SMMLV); en orden ascendente, el siguiente es de \$48.978.256,oo; el tercero alcanza los $\$ 61.222 .820$,oo.

\subsubsection{Programas a distancia}

- Pregrado. 23 programas: 11 ciclo profesional y 12 tecnologías. Se destacan cuatro nuevos programas. El valor de las carreras, según la duración y el del semestre, sería el siguiente: 10 semestres $\$ 7.929 .752$,oo (un salario mínimo mensual legal vigente); 1,25 \$9.912.192,oo; 1,5 \$11.894.628,oo; 2,0 \$15.859.504,oo; y 2,5 $\$ 19.824 .380,00)$. 
- Posgrados. Los posgrados del programa a distancia son los siguientes: Especialización en Alta Gerencia de Empresas, Especialización en Didáctica de la Matemática para la Educación Básica; Especialización Gerencia de Empresas de Salud; Maestría en Dirección y Administración de Empresas. El valor de cada semestre sería de: 4,5 SMMLV \$3.102.548 (total estudios $\$ 12.844 .548$, oo) y el de 12 SMMLV \$8.273.460,oo (total estudios \$18.944.568,oo). La duración de cada uno de los programas es de cuatro semestres.

Se concluye que la privatización de la educación superior, mediante los diferentes programas que brinda la UPTC, especialmente los presenciales (pregrado, posgrado, maestrías y doctorados) y mínimamente en los virtuales, se acentuó a partir del año 2010, cuando se modificaron los criterios socioeconómicos de la población, incluyendo otros que permitieron el incremento de estos valores en forma desbordada, acentuando la crisis para las personas de menos recursos que cada vez ven más lejana la posibilidad de culminar una carrera profesional.

\subsection{Discusión de resultados}

De las siete transformaciones expuestas por Roth (2011), seis de ellas reflejan los avances significativos que ha tenido la educación superior, y la séptima, la "privatización-mercantilización" de la misma, porque el Estado se olvidó del cumplimiento de uno de sus deberes contemplados en la Constitución Política de Colombia y, por el contrario, propició su funcionamiento al "modelo de gestión empresarial", priorizando la rentabilidad sobre la calidad. En el mismo sentido, la privatización también se orientó a la búsqueda de nuevas fuentes de recursos del capital privado, lo que imposibilita que la eficiencia en los contenidos sea una prioridad.

Harris (2009), refiriéndose a las transformaciones de la educación superior, aduce que los cambios, avances tecnológicos y la globalización están interconectados, y es lo que genera la modificación de derechos sociales (educación y la salud) para considerarlos como servicios, los cuales requieren de autofinanciamiento, que incide en la mercantilización de estas. Afectan, de la misma manera, las relaciones entre educación superior, entorno y el futuro de la sociedad, especialmente la de aquellos miembros que están ubicados en los estratos medio y bajos (Harris, 2009).

Además, si Colombia invirtiera los recursos promedio que se usan para los pilos ( $\$ 18$ millones año, incluye sostenimiento y otros) en los estudiantes que hoy acceden a la educación superior en IES oficiales (1.045.200 en el año 2014), tendría que incrementar el presupuesto de la educación superior 6 veces; es decir, pasar de $\$ 3$ billones a $\$ 18$ billones anuales. Lo anterior explica las condiciones, los resultados de calidad y el escaso impacto regional que tiene la mayoría de IES oficiales, así como de alguna manera por qué los pilos prefieren las universidades privadas (Pérez, 2016). 


\section{Planeación}

\subsection{Propuesta}

El Estado colombiano ha elaborado una guía para la elaboración de políticas públicas, cuyos parámetros son tenidos en cuenta solo de manera parcial, que ante la falta de compromiso político, no se diseñan, ajustan y financian, por las exigencias de los organismos que conceden los recursos de la deuda en dólares (FMI, BM, BID). Entre los principales aspectos están: a) principios orientadores; b) estrategia que contribuya al desarrollo del país; c) regionalización, d) funcionamiento sistémico, e) educación superior como bien público, f) diferenciación y g) calidad.

\section{Gestión}

\subsection{Conclusiones}

Las organizaciones internacionales, en el contexto globalizante de hoy (2016), intervienen activamente en las políticas educativas del país, por encima de los actores históricos de este (Estado y sociedad civil); un ejemplo de ello es el programa PISA, que evalúa la calidad de la educación y determina cuál es la mayoría con educación de baja calidad y la minoría que muestra estándares que los satisfacen.

Las directrices dictadas por estas organizaciones se orientan hacia la elaboración, evaluación y financiación de las políticas públicas, enfatizando más en la última, como consecuencia de las dos anteriores, porque a través de la imposición de gravámenes y privatización de lo social, se garantiza el pago del capital e intereses de la creciente deuda externa (en dólares). En este caso, las IES que imparten la educación superior se han visto afectadas, pues ante la disminución del apoyo económico estatal, decidieron para mantenerse en el mercado nacional y hacerle frente al monstruo de la internacionalización, aumentar el valor de los semestres, especialmente de posgrado, doctorados y servicios para los estudiantes, desconociendo en gran manera, la oportunidad que se le venía brindando a jóvenes con bajos recursos económicos, $\mathrm{y}$, por lo tanto, violando el derecho fundamental de la educación.

La vulneración del derecho a la educación por parte de las políticas educativas en Colombia se viene presentando desde el año 1992, con la Ley 30, reflejada en los diversos planes de desarrollo, donde los programas de gobierno de cada mandatario se orientaron a disminuir los recursos que aportaba el Estado, incluyendo los subsidios con que se beneficiaban muchos estudiantes de bajos recursos. En este caso, a diferencia de otras acciones y proyectos que requerían continuidad, para su logro sí hubo continuidad, como es el caso de la UPTC, para degradar su condición de pública. 
Por otra parte, los organismos de crédito, proveedores de recursos a través de la deuda externa, exigían y siguen exigiendo condiciones de privatización para la educación superior, con la excusa de poder cumplir los compromisos económicos adquiridos, los cuales van mucho más allá de tales propósitos, al imponer condiciones que acrecientan la dependencia. Respecto a la internacionalización, producto de la globalización, la UPTC intenta emular el ambiente que se impone en centros educativos de otros países desarrollados, en cuanto a calidad educativa, evaluación, portafolio de programas, entre otros.

\section{Referencias}

Altbach, P. et al. (20 de marzo de 2010). Tras la pista de una revolución académica: Informe sobre las tendencias actuales. En Conferencia Muncial sobre la Educación Superior. Recuperado de http://portal.unesco.org/education/es/ev.php-

Asociación Colombiana de Universidades (ASCUN). (2008, 11 de ago.). Propuestas $y$ acciones universitarias para la transformación de la educación superior en América Latina. Recuperado de http://www.6x4uealc.org/site2008/pre/6x4_prec. pdf

Asociación Colombiana de Universidades (Ascún) (2014). Problemas nodales de la internacionalización de la educación superior en Colombia, principios orientadores y lineamientos para la construcción de política pública. Bogotá: Ascún.

Asociación Internacional de Universidades. (2012). Reforzar los valores académicos en la internacionalización de la educación: una llamada a la acción. México: IAU.

Banco Mundial. (1998). La educación superior en América Latina. Nueva York: Banco Mundial.

Banco Mundial. (2002). Proyect Appraisal Document on a Proprosed Loan in the Amount of US\$200 Million to the Republic of Colombia for a Higher Education. Washington, D.C.: Banco Mundial.

Bank for Reconstruction and Development/The World Bank. (2012). Evaluaciones de políticas nacionales de educación: la educación superior en Colombia 2012. Nueva York: Bank for Reconstruction and Development/The World Bank.

Cañón, J. (2005). Internacionalización de la educación superior y educación superior internacional: elementos para un análisis sociológico general. Revista Colombiana de Sociología, (25), 105-125.

Castell, S. (1996). La era de la información. Madrid: Alianza. 
Chiroleu, A. \&. (2005). La reforma de la educación superior como capítulo de la reforma del Estado. Peculiaridades y trazos comunes. Buenos Aires: Prometeo Libros, Universidad Nacional de General Sarmiento.

De Wit, H. (Ed.). (2013). An Introduction to Higher Education Internationalisation. Milan: Universita Cattolica University Press Vita e Pensiero, Centre for Higher Education Internationalisation (CHEI), UCSC.

Driessnack, M. e. (2007). Revisión de los diseños relevantes para la enfermería: parte 3: métodos mixto y múltiples. Revista Latinoamericana de Enfermería, 15(15), 179-182.

Harris, S. (2009). Repensando las identidades académicas en los tiempos neoliberales. Madrid: Plaza y Valdés.

International Association of Universities. (2012). Reforzar los valores académicos en la internacionalización de la educación superior: una llamada a la acción. París: International Association of Universities.

Johnson, B. \& Onwuegbuzie, A. (2004). Los métodos de investigación mixtos: un paradigma de investigación cuyo tiempo ha llegado. Educational Researcher $33(7), 14-26$.

Ministerio de Educación Nacional. (2013). Diálogos regionales sobre educación superior. Bogotá: MEN.

Misas, G. (2004). La educación superior en Colombia. Análisis y estrategias para su desarrollo. Bogotá: Universidad Nacional.

Nussbaum, M. (2010). Sin fines de lucro. Por qué la democracia necesita de las humanidades. Buenos Aires: Katz.

Organización para la Cooperación y el Desarrollo Económico (OCDE). (2012). Education at a Glance 2012. París: OCDE.

Pérez, A. (2016, 2 de jul.). Los privilegios de ser pilo paga. Recuperado de http://www. universidad.edu.co/index.php?option $=$ com_content\&view $=$ article $\&$ id $=7033$ :losprivilegiados-de-ser-pilo-paga\&catid=12:opini\&Itemid $=200$

Ramírez, A. (2012). Condiciones para la internacionalización de la educación superior: entre la inclusión y la exclusión en un mundo globalizado. Revista de Universidad y Sociedad del Conocimiento, 8(2), 152-164.

Roth, A. (2011). Los procesos de cambios en las políticas públicas de educación superior en América Latina desde una perspectiva comparada. Bogotá: Universidad Nacional, Programa Interdisciplinario en Política Educativas (PIPE). 
Universidad Nacional. (2009). Metodología para el cálculo del valor de las matrículas en la Universidad Pedagógica y Tecnológica de Colombia de Colombia (UPTC). Bogotá: Universidad Nacional.

Universidad Pedagógica y Tecnológica de Colombia (UPTC). (2015, 30 de dic.). Derechos complementarios. Recuperado de http://www.uptc.edu.co/admisiones/ valores/2016.

Universidad Pedagógica y Tecnológica de Colombia (UPTC). (2016, 10 de marzo). Programas de pregrado a distancia. Recuperado de http://www.uptc.edu.co/ vicerectoria_academica/programas/adistancia/inf_general/index.html\#

Van der Wende, M. (2001). Internationalisation Oolicies: About New Trends and Contrasting Paradigms. Enschede, Nettherlands: Higher Education Policy.

Vásquez, O. (2011). La internacionalización de la educación superior en Colombia. Medellín: Asociación de Profesores de la Universidad de Antioquia.

Yin, R. (1994). Case study research: Design and Methods. Thousand Oaks: Sage Publications. 